\title{
Effects of exposure of rat erythrocytes to a hypogeomagnetic field
}

A.D. Nadeev ${ }^{\text {a,b }}$, M.A. Terpilowski ${ }^{\text {a }}$,V.A. Bogdanov ${ }^{\text {c }}$, D.A. Khmelevskoy ${ }^{\text {a }}$, B.F. Schegolev

d,e, S.V. Surma ${ }^{\text {d }}$, V.E. Stefanov ${ }^{\text {c }}$, R.O. Jenkins ${ }^{\text {f }}$ and N.V. Goncharov ${ }^{\text {a,g,* }}$

${ }^{a}$ Sechenov Institute of Evolutionary Physiology and Biochemistry, St. Petersburg, Russia

${ }^{\mathrm{b}}$ Institute of Cell Biophysics, Pushchino, Russia

c St. Petersburg State University, St. Petersburg, Russia

${ }^{\mathrm{d}}$ Pavlov Institute of Physiology, St. Petersburg, Russia

e Almazov National Medical Research Centre, St. Petersburg, Russia

${ }^{\mathrm{f}}$ School of Allied Health Sciences, De Montfort University, Leicester,UK

g Research Institute of Hygiene, Occupational Pathology and Human Ecology, Kuz’molovsky Russia

* Corresponding author: Nikolay V. Goncharov, Sechenov Institute of Evolutionary

Physiology and Biochemistry, Russian Academy of Sciences, pr. Toreza 44, St. Petersburg, 194223 Russia, email: ngoncharov@gmail.com

Published by IOS Press; https://www.iospress.nl/journal/biomedical-spectroscopy-andimaging/

NADEEV AD, TERPILOWSKI MA, BOGDANOV VA, KHMELEVSKOY DA, SCHEGOLEV BF, SURMA SV, STEFANOV VE, JENKINS RO and GONCHAROV NV. Effects of exposure of rat erythrocytes to a hypogeomagnetic field. Biomedical Spectroscopy and Imaging. 2018: June $19^{\text {th }}$,Pre-press, pp. 1-9, DOI: 10.3233/BSI180181 


\section{Abstract}

BACKGROUND: Hypomagnetic fields can disrupts the normal functioning of living organisms by a mechanism thought to involve oxidative stress. In erythrocytes, oxidative stress can inter alia lead to changes to hemoglobin content and to hemolysis.

OBJECTIVE: To study the effects of hypomagnetism on the state of rat erythrocytes in vitro. METHODS: Rat erythrocytes were exposed to an attenuated magnetic field (AMF) or Earth's magnetic field (EMF), in the presence of tert-butyl hydroperoxide (TBHP) as inducer of oxidative stress. Determinations: total hemoglobin (and its three forms - oxyhemoglobin, methemoglobin, and hemichrome) released from erythrocytes, spectral data (500-700nm); oxygen radical concentrations, electron paramagnetic resonance.

RESULTS: AMF and EMF exposed erythrocytes were compared. After 4h incubation at high TBHP concentrations (>700 $\mu \mathrm{M})$, AMF exposed erythrocytes released significantly more $(\mathrm{p}<0.05)$ hemoglobin $(\mathrm{Hb})$, mostly as methemoglobin (metHb). Conversely, after $24 \mathrm{~h}$ incubation at low TBHP concentrations $(\leq 350 \mu \mathrm{M})$, EMF exposed erythrocytes released significantly more $\left(p^{<0.001)}\right.$ hemoglobin, with metHb as a significant proportion of the total Hb. Erythrocytes exposed to AMF generated more radicals than those exposed to the EMF. CONCLUSION: Under particular conditions of oxidative stress, hypomagnetic fields can disrupt the functional state of erythrocytes and promote cell death; an additive effect is implicated.

Keywords: erythrocytes, haemoglobin, hypomagnetic field, reactive oxygen species, cell death 


\section{Introduction}

All living organisms experience the effect of the Earth's magnetic field (EMF), which varies depending on the degree of latitude increasing to the poles and averaging $42 \mu \mathrm{T}$ [1]. The magnitude of the induction of the magnetic field in near-Earth space is much lower than the magnitude of the magnetic field on the surface of the Earth and amounts to $0.0066 \mu \mathrm{T}$ in interplanetary space, $0.3 \mu \mathrm{T}$ at the lunar surface, $\sim 0.7 \mu \mathrm{T}$ at an altitude of $200 \mathrm{~km}$ from the surface of Mars [2-4].

Research progress into the biological effects of hypomagnetic fields has recently been reviewed [5]. Such research has potential relevance to the health and wellbeing of astronauts during aerospace travel and space station living. In a number of experiments it was shown that the use of a static magnetic field attenuated by screening (AMF) with induction of less than $20 \mu \mathrm{T}$ violates the functional state of living organisms [6-9]. AMF can affect both the rate and duration of the cell cycle, and the effects of exposure to AMF on human lymphocytes are more significant in the G1 phase [10, 11]. Reducing the induction of the EMF to $300 \mathrm{nT}$ leads to inhibition of proliferation and differentiation of skeletal muscle cells in newborn rats [9, 12].

It is assumed that the mechanism of the action of weak magnetic and electromagnetic fields in biological systems is associated with the generation of reactive oxygen species (ROS) $[13,14]$. On the other hand, it has been found that intracellular production of hydrogen peroxide $\left(\mathrm{H}_{2} \mathrm{O}_{2}\right)$ in cancer cells and in endothelial cells of arteries is suppressed under AMF $[15,16]$. It is also assumed that the geomagnetic sensitivity of living organisms depends on the generation of the superoxide anion $\left(\mathrm{O}^{2 \cdot}\right)[17,18]$. Hypomagnetic fields can modulate the interconversion of a singlet-triplet transition involving superoxide [18-20].

Generation of ROS by erythrocytes induces autoxidation, the essence of which is spontaneous oxidation of heme iron $\left(\mathrm{Fe}^{2+}\right)$. This process, if not controlled by the antioxidant defense system, leads to the formation of metHb $\left(\mathrm{Fe}^{3+}\right)$ and $\mathrm{O}^{2 \cdot}[21]$. Superoxide can react 
with hemoglobin ( $\mathrm{Hb})$ and undergo dismutation with the formation of $\mathrm{H}_{2} \mathrm{O}_{2}$, which can initiate lipid peroxidation and/or react with $\mathrm{Hb}$ [22]. If the antioxidant system of erythrocytes is disrupted, too much ROS are produced, oxidative stress leads to the destruction of red blood cells (hemolysis), $\mathrm{Hb}$ is released into the intravascular space and endothelial NO is consumed, resulting in spasm and hypertension [23]. In addition, oxidative stress causes increased erythrocyte rigidity as a result of eryptosis, which can lead to anemia and / or blood microcirculation disruption [23].

The purpose of the research reported on here was to study the effects of AMF on the state of rat erythrocytes in vitro.

\section{Materials and methods}

\subsection{Reagents}

HEPES and TEMPO (4-amino-2,2,6,6-tetramethylpiperidine-1-oxyl) were from Sigma (USA), all other reagents were from Vecton (Russia).

\subsection{Preparation of red blood cells}

Heparinized blood of Wistar rats was used. All manipulations with the animals were performed in accordance with the National Institutes of Health guide for the care and use of Laboratory animals (NIH Publications No. 8023, revised 1978). Blood obtained after decapitation was centrifuged for $3 \mathrm{~min}$ at $3000 \mathrm{rpm}$ and the plasma and lymphocyte-platelet layer were taken. The remaining erythrocyte mass was resuspended in $\mathrm{Ca}^{2+}$-HEPES buffer (FC in mM: $140 \mathrm{NaCl}, 3 \mathrm{MgCl}_{2}, 10$ Glucose, $10 \mathrm{HEPES}, 2.5 \mathrm{CaCl}_{2}$ and $5 \mathrm{KCl}$ ) pH 7.4 and centrifuged twice for 2 minutes at $2000 \mathrm{rpm}$. One volume of $\mathrm{Ca}^{2+}$-HEPES buffer was added to the washed red blood cells, resuspended and blood counts were performed on a hematologic analyzer (Medonic M16). The number of lymphocytes in the suspension was $<0.1 \times 10^{9} / \mathrm{L}$, and platelets $<0.01 \times 10^{9} / \mathrm{L}$. The resulting suspension was adjusted to an erythrocyte concentration of $0.510^{12} / \mathrm{L}$ using $\mathrm{Ca}^{2+}$-HEPES buffer and poured into test tubes, which were subsequently used in the work. tert-Butyl hydroperoxide (TBHP) was used to induce 
oxidative stress. A working solution of TBHP was obtained by diluting a stock 7.7 M solution just before the experiment. The test tubes were incubated on an orbital shaker (BioSan, OS20) at $100 \mathrm{rpm}$ for 4 and $24 \mathrm{~h}$. During incubation the tubes were kept in a horizontal position at a room temperature of $24{ }^{\circ} \mathrm{C}$ with exposure to EMF or AMF.

\subsection{Determination of hemoglobin forms}

After incubation some of the samples were transferred into separate tubes and centrifuged at $3000 \mathrm{rpm}$. A spectrum was obtained from each supernatant at wavelengths from 500 to 700 nm with $1 \mathrm{~nm}$ step in a cuvette of $0.8 \mathrm{~cm}$ width. Spectrum data were used to calculate the molar concentration of total hemoglobin and its three forms - oxyhemoglobin (oxyHb), methemoglobin (metHb) and hemichrome (HC) - using the appropriate molar extinction coefficients [24]. The Hb concentration was expressed in $\mu$ mol per $0.2 \mathrm{ml}$ of erythrocyte suspension at a concentration of $5 \cdot 10^{11} / \mathrm{L}$.

\subsection{Determination of oxygen radical concentration by a spin-trap method using electron paramagnetic resonance (EPR)}

The production of ROS in cells after exposure to EMF and to AMF was investigated by adding 4-amino-2,2,6,6-tetramethylpiperidine-1-oxyl (TEMPO) as a spin-trap at a concentration of $0.3 \mathrm{mM}$ to the erythrocyte suspension before incubation. After 15 hours of incubation, the samples were frozen in liquid nitrogen. The spectra of the obtained lysate of the erythrocyte suspension were recorded on an EPR spectrometer (Bruker Elexsys E580), designed to study solids (including single crystals), liquids and aqueous solutions at $24.2{ }^{\circ} \mathrm{C}$ (297.2 K) with slow scanning (16 scans) in 30 s and 5 s wait before re-scanning.

Electron spin resonance (ESR) spectroscopy combined with a spin-trap method is based on the idea that certain compound with a molecular structure quite close to that of nitroxyl radical (spin-trap) is connecting with free short-living radical (e.g. hydroxyl radical, ${ }^{-O H}$ ) forming a long-living nitroxide radical (spin inclusion complex). The EPR spectrum for this new stable complex is unique for this radical or for the family of such radicals. TEMPO is one of the 
most extensively used spin-trap compounds since the 1960s [25]. It is difficult to quantify the amount of ROS in the intact biological system, because they are unstable and rapidly dismutate or react with adjacent molecules. ESR combined with a spin-trap such as TEMPO is a reliable, direct and fast method for measuring ROS. Nitroxide radical is a stable chemical and can be easily detected by ESR within biological tissues in vitro and in vivo [26, 27]. In the present research, ESR investigation is aimed at the ${ }^{-} \mathrm{OH}$ detection.

\subsection{Attenuation of the Earth's magnetic field in the shielding chambers}

For physical modeling of an ultraweak static magnetic field, two shielding chambers were created in the form of cylinders $\left(1^{\text {st }}\right.$ chamber: diameter $=10 \mathrm{~cm}$, length $=30 \mathrm{~cm}, 2^{\text {nd }}$ chamber: diameter $=25 \mathrm{~cm}$, length $=60 \mathrm{~cm}$ ), covered with tens of layers of shielding material manufactured from alloys of amorphous soft magnetic material AMAG172 (Kuznetsov et al., Russian Patent No: No. 2324989, 2008). All MF measurements were carried out using a Fluxmaster magnetometer (Stefan Mayer Instruments, Dinslaken, Germany) in the range of 1 nT - $200 \mu \mathrm{T}$ with a resolution of $1 \mathrm{nT}$, and with magnetometer (HB0302.1A, St. Petersburg, Russia) in the range $0.1-100 \mathrm{mT}$ with resolution of $0.1 \mathrm{mT}$.

\subsection{Statistics}

Determination of hemoglobin forms was performed as 10 independent experiments. Blood was derived from one rat in each experiment $(n=10)$ and split into several samples which were exposed to either EMF or AMF. Three parallel measurements of each of the $\mathrm{Hb}$ forms, which were further averaged, were done per sample. Thus, each group (EMF or AMF) included 10 averaged measurements per Hb form. The statistical analysis was done using GraphPad Prism 7 (GraphPad Software, Inc., San Diego, CA). Data were analyzed using paired Student's t-test, and Benjamini-Krieger-Yekutieli false discovery rate correction procedure was applied [28].EPR spectra were further quantified using calculation of energy and average power over finite magnetic field strength interval. This computation was done 
using spline (degree $=3$ ) curve fitting to obtained spectra and subsequent integration (and normalization by magnetic field strength for power calculation).

\section{Results}

\subsection{Hemoglobin in the supernatant of erythrocytes after exposure to EMF or AMF}

Estimation of total Hb showed that samples incubated in the presence of relatively low concentrations of TBHP (up to $350 \mu \mathrm{M}$ ) for $4 \mathrm{~h}$ had released the same quantity of Hb while exposure to either the EMF or AMF (Fig. 1a). However, at high concentrations of TBHP (above $700 \mu \mathrm{M}$ ) samples incubated for 4 h released less Hb under EMF exposure than AMF exposed samples; Hb release was generally lower by nearly $12 \%$ with significant difference $(\mathrm{p}<0.05)$ for all concentrations (Fig. 1a). Conversely, after $24 \mathrm{~h}$ incubation, in samples that were incubated with low TBHP concentrations (up to $350 \mu \mathrm{M}$ ) under EMF the total $\mathrm{Hb}$ released was significantly higher (from 74 to $316 \%, \mathrm{p}<0.001$ ) compared to the supernatant of samples that were incubated with exposure to AMF (Fig. 1b). In the presence of high concentrations of TBHP (above $700 \mu \mathrm{M}$ ), Hb release in samples exposed to AMF was generally higher but the difference was insignificant.

Determination of three forms of $\mathrm{Hb}$ showed that after $4 \mathrm{~h}$ of incubation, the difference in $\mathrm{Hb}$ released for samples with high TBHP concentrations (above $500 \mu \mathrm{M}$ ) was mostly in the metHb form (approximately 52 to $72 \%$ of total $\mathrm{Hb}$ concentration). After $24 \mathrm{~h}$ of incubation, the difference in $\mathrm{Hb}$ released for samples with low TBHP concentrations (up to $350 \mu \mathrm{M}$ ) was mostly in the oxyHb form (46 to $68 \%$ of total $\mathrm{Hb}$ concentration), though metHb consisted a significant part of the total Hb. Interestingly, in the case of spontaneous oxidation of erythrocytes in the absence of TBHP, after $24 \mathrm{~h}$ of incubation the release of total $\mathrm{Hb}$ from cells incubated with exposure to AMF was 162\% lower than for cells incubated with exposure to EMF $(p<0.01)$ (Fig. 2a). The amounts of metHb and oxyHb in the supernatant of the samples after incubation with exposure to EMF were 457\% (p <0.001) and 198\% (p <0.05) higher, respectively, compared to those for AMF exposed samples (Fig. 2b and 2c). 


\subsection{The level of oxygen radicals in the supernatant of erythrocytes after exposure to EMF}

or $A M F$

Measurement of the relative amounts of oxygen radicals (mainly the hydroxyl radical and superoxide anion), by EPR using the TEMPO spin label, showed that in the absence of TBHP after $24 \mathrm{~h}$ incubation there was a significant decrease in the intensity of the spectrum for the lysate of the erythrocyte suspension under exposure to AMF, compared to that under exposure to EMF (Fig. 3a). This indicates that in erythrocytes exposed to AMF, a greater number of free radicals are formed than under normal EMF conditions. Conversely, however, in the presence of TBHP (100 and $250 \mu \mathrm{M})$, after $24 \mathrm{~h}$ incubation the increase in the intensity of the spectrum for the lysate from AMF exposed erythrocytes was more than that for the lysate from EMF exposed erythrocytes (Fig. 3b and 3c). In general, there was a dose-related increase of the intensity of label spectrum with increase in TBHP concentration (Fig.4). Paradoxically it may seem, but this clearly indicates that TBHP at relatively low doses suppressed oxygen radical formation in the erythrocyte suspension.

\section{Discussion}

We did not detect differences in the effect of relatively low TBHP concentrations after 4 hours under the AMF or EMF conditions. Similarly, there was no difference in the effect of high concentrations of TBHP after 24 hours. An elevated release of total Hb - mainly as metHb - after 4 hours of incubation under AMF conditions in comparison with EMF, in the presence of high concentrations of TBHP, indicates an increase in cell lysis, i.e. necrotic death of erythrocytes and, consequently, potentiation of the action of TBHP by the attenuated geomagnetic field. An elevated release of total $\mathrm{Hb}$ after $24 \mathrm{~h}$ of incubation under AMF conditions in comparison with EMF, in the presence of high concentrations of TBHP, is less prominent and insignificant, which is explained by the time-dependent fulfillment of the entire process. The most interesting phenomenon is elevation of Hb under conditions of EMF, both 
in the absence and low concentrations of TBHP, when the oxyHb form slightly predominates, with metHb representing a significant part of the total Hb. In accordance with this, TBHP at relatively low doses suppressed oxygen radical formation in the erythrocyte suspension.

In our recent in vivo study on rats [29], there was no apparent effect of AMF on hematological parameters and, of particular note, there was no effect on the number and average volume of erythrocytes or the concentration of Hb within them. This does not imply the absence of the effect of AMF on the biochemistry of erythrocytes in the longer term, but may indicate either an ability through hematopoiesis to quickly replace damaged cells in the compensatory phase and/or the absence of manifestations of a weak effect of AMF on the hematological parameters within the limited time of the in vivo experiment. In addition, the absence of a visible AMF effect under the usual conditions of the animals existence does not mean that under the conditions of oxidative or other types of stress, the effect of AMF would not be manifested much earlier and stronger. Additivity, synergy or potentiation of different effects - acting stronger or weaker on various targets - could amplify the effect of AMF. Therefore, in recognition of the inherent limitations of an in vivo rat model, in the present work we developed an in vitro model to detect a possible effect of AMF on erythrocytes within a reasonable time frame. It should be pointed out in this context that no evidence for damage of erythrocytes was obtained in the previous in vivo experiments, due to application of generally available and lowspecific methods of clinical biochemistry. After obtaining the novel in vitro data from the present research it is now pertinent to consider development of a novel methodology that could reveal slight shifts in the state of red blood cells. This is especially important in view of the need to monitor the health and wellbeing of astronauts during aerospace travel.

In the context of aerospace travel, arguments could be given against the use of rat erythrocytes instead of human erythrocytes in experimentation. Indeed, there are differences in membrane properties of erythrocytes in these two species, such as erythrocyte deformability, cell membrane stability, osmotic gradient ektacytometry (osmoscan) and aggregation tendency 
[30-32]. Furthermore, human erythrocyte membranes have comparatively lower total phospholipid and cholesterol contents, contain lower proportions of acidic phospholipids and higher proportions of lysophosphoglyceride and sphingomyelin, and are relatively more fluid; some of these properties have been shown to correlate well with the lower $\mathrm{V}_{\max }$ of the $\mathrm{Na}^{+} / \mathrm{K}^{+}$ATPase of human RBC membranes [31]. Despite these differences, similar results have been obtained in studies of aging, antioxidant nutraceuticals etc. upon this ATPase and other enzymes and transporters of rat and human erythrocytes [33-36]. Since our previous in vivo experiments under AMF conditions was with rats [29], it was logical for the present in vitro research to continue the experimentation with rat RBC.

The method we applied here for assessment of ROS generation provides several advantages. Firstly, it allows direct quantitative assessment of ROS, which is not possible with other techniques. Secondly, this method does not require complicated subcellular fractions procedures, which may alter the status of ROS. ESR can also measure the net amount of ROS even in the presence of antioxidants. Among the shortcomings of the ESR method we have to mention non-resonance absorption of the microwave frequency by biological objects, which can provoke heating of the sample. In order to prevent this process we applied a special thermostating. In addition, ESR is a rather expensive and labour-intensive technique so future research should focus on its simplification, together with elaboration of alternative techniques such as calculation of complex non-invasive biomarkers based on single and simple physiological and/or biochemical indicators.

Exposure of the living cells to high concentrations of TBHP results in a significant increase in cytotoxicity, ROS generation and lipid peroxidation. TBHP generates tert-butoxyl $(t-\mathrm{BuO} \bullet)$ and tert-butyl peroxyl (t-BuOO•) radicals. The oxidative processes induced by TBHP in erythrocytes lead to free radicals formation in the reaction between TBHP and cellular $\mathrm{Hb}$, which are then immediately consumed in lipid peroxidation reactions [37]. In addition, high concentrations of TBHP (0.5 mM) cause a decrease in activity of catalase and SOD [38]. The antioxidant/prooxidant 
effects are not only correlated with the molecular structure but also the distributive status in the reaction system [39]. From this point of view, one should take into consideration that the redox potential $\Delta \xi^{\circ}$ of TBHP $\left(t-\mathrm{BuO}_{2}{ }^{\circ} / t-\mathrm{BuO}_{2} \mathrm{H}\right.$ redox pair) is at the level of $1050 \mathrm{mV}$ and $\mathrm{LogP}$ is about $1.23[40,41]$. This means that at low concentrations of TBHP, this oxidant can accept electrons from oxygen radicals such as superoxide anion and hydrogen peroxide ( $\Delta \xi^{\circ \prime}$ of this redox pair is about $360 \mathrm{mV}$ [42]) in the hydrophobic phase, protecting the membrane of erythrocytes from being oxidized. Even in the case of slow development of prooxidant effects, they initiate development of eryptosis with rigid membranes not prone to be broken easily, as we observed after $24 \mathrm{~h}$ exposure to low doses of TBHP.

The mechanism of amplification of oxidative stress in AMF conditions can be explained in terms of cytotoxic power, which assumes calculation of the rate of development of cytotoxic effects per unit of time [43]. Superposition of the cytotoxic effect of high power (TBHP in high concentration) causing necrotic type of cell death, with the low cytotoxic effect of AMF exposure inducing apoptotic cell death, leads to an intensification and acceleration of the necrotic pathway. While the superposition of two relatively low-power influences, each inducing apoptotic pathways, primarily accelerates the development of apoptosis. To clarify the molecular mechanisms of interaction between TBHP and AMF, it is necessary to estimate the level of reduced glutathione, the activity of the main antioxidant protection systems of erythrocytes, the activity of NADPH oxidase and the system of transmembrane electron transport. These aspects are the foci of our further research.

\section{Acknowledgements}

These studies were supported and by the Russian FASO (programme AAAA-A18118012290142-9) and by the Program of Fundamental Scientific Investigations of State Academies for 2014-2020 (SP-14, division 63). 


\section{References}

[1] C.C. Finlay, S. Maus, C.D. Beggan, T.N. Bondar, A. Chambodut, T.A. Chernova, A. Chulliat, V.P. Golovkov, B. Hamilton, M. Hamoudi, R. Holme, G. Hulot, W. Kuang, B. Langlais, V. Lesur, F.J. Lowes, H. Lühr, S. Macmillan, M. Mandea, S. McLean, C. Manoj, M. Menvielle, I. Michaelis, N. Olsen, J. Rauberg, M. Rother, T.J. Sabaka, A. Tangborn, L. Tøffner-Clausen, E. Thébault, A.W.P. Thomson, I. Wardinski, Z. Wei and T.I. Zvereva, International geomagnetic reference field: the eleventh generation, Geophysical Journal International 183(3) (2010), 1216-1230.

[2] F. Steinhilber, J.A. Abreu, J. Beer and K.G. McCracken, Interplanetary magnetic field during the past 9300 years inferred from cosmogenic radionuclides, Journal of Geophysical Research 115 (2010), 1-14.

[3] M.S. Berguig, M. Hamoudi and J.L. Lemoueel, Validate global mapping of internal lunar magnetic field. Arabian Journal of Geoscience 6(4) (2011), 1063-1072.

[4] T.R. Watters, P.J. McGovern and R.P. Irwin III, Hemispheres apart: the crustal dichotomy on mars, Annual Review of Earth Planetary Science 35 (2007), 621-652.

[5] J. Ouyang and H. Li, Biological effects due to hypomagnetic field and its research progress. Journal of Life Science Research 4(2) (2017), 14-19.

[6] A.P. Dubrov, The geomagnetic field and life: geomagnetobiology, Plenum, New York (translated from Russian by F.l. Sinclair), 1978.

[7] N.A. Belyavskaya, Biological effects due to weak magnetic field on plants, Advances in Space Research 34 (2004), 1566-1574.

[8] B. Jia and P. Shang, Research progress of biological effects of hypomagnetic fields, Space Medicine \& Medical Engineering 22 (2009), 308-312.

[9] S.V. Surma, G.B. Belostotskaya, B.F. Shchegolev and V.E. Stefanov, Effect of weak static magnetic fields on the development of cultured skeletal muscle cells. Bioelectromagnetics 35(8) (2014), 537-546. 
[10] C.F. Martino, H. Perea, U. Hopfner, V.L. Ferguson and E. Wintermantel, Effects of weak static magnetic fields on endothelial cells, Bioelectromagnetics 31 (2010), 296-301.

[11] I.Y. Belyaev, Y.D. Alipov and M. Harms-Ringdahl, Effects of zero magnetic field on the conformation of chromatin in human cells, Biochimica et Biophysica Acta 1336 (1997), 465473.

[12] I.S. Eldashev, B.F. Shchegolev, S.V. Surma and G.B. Belostotskaia, Effect of lowintensity magnetic fields on the development of satellite muscle cells of a newborn rat in the primary culture, Biofizika 55 (2010), 868-874.

[13] I.M. Spivak, M.L. Kuranova, G.R. Mavropulo-Stolyarenko, S.V. Surma, B.F.

Shchegolev and V.E. Stefanov, Cell response to extremely weak static magnetic fields, Biophysics 61(3) (2016), 435-439.

[14] A.L. Buchachenko and R.G. Lawler, New possibilities for magnetic control of chemical and biochemical reaction, Accounts of Chemical Research 50(4) (2017), 877-884.

[15] C.F. Martino and P.R. Castello, Modulation of hydrogen peroxide production in cellular systems by low level magnetic fields, Plos One 6(8) (2011), e22753

[16] C.F. Martino, L. Portelli, K. Mccabe, M. Hernandez and F. Barnes, Reduction of the earth’s magnetic field inhibits growth rates of model cancer cell lines, Bioelectromagnetics 31 (2010), 649-655.

[17] T. Ritz, R. Wiltschko, P.J. Hore, C.T. Rodgers and K. Stapput, Magnetic compass of birds is based on a molecule with optimal directional sensitivity, Biophysical Journal 96 (2009), 3451-3457.

[18] I.A. Solov’yov, and K. Schulten, Magnetoreception through cryptochrome may involve superoxide, Biophysical Journal 96 (2009), 4804-4813.

[19] T. Ritz, S. Adem and K. Schulten, A model for photoreceptor-based magnetoreception in birds, Biophysical Journal 78 (2000), 707-718. 
[20] R.J. Usselman, C. Chavarriaga, P.R. Castello, M. Procopio, T. Ritz, E.A. Dratz, D.J. Singel and C.F. Martino, The quantum biology of reactive oxygen species partitioning impacts cellular bioenergetics, Scientific Reports 6 (2016), 1-6.

[21] P.H. Misra and I. Fridovich, The generation of superoxide radical during the autoxidation of hemoglobin, Journal of Biological Chemistry 10 (1972), 6960-6962.

[22] C. Giulivi and E. Cadenas, Heme protein radicals: formation, fate, and biological consequences, Free Radical Biology and Medicine 24 (1998), 269-279.

[23] E. Lang and F. Lang, Mechanisms and pathophysiological significance of eryptosis, the suicidal erythrocyte death, Seminars in Cell and Developmental Biology 39 (2015), 35-42. [24] I.I. Stepuro, N.A. Chaikovskauya, V.P. Vodoevich and V.V. Vinogradov, Reduction of met-hemoglobin and ferricytochrome c by glycosylated amino acids and albumin, Biochemistry 62(9) (1997), 967-972.

[25] O.L. Lebedev and S.N. Kazarnowskii, Catalitic oxidation of aliphatic amines with hydrogen peroxide, Zhur. Obshch. Khim. 30 (5) (1960), 1631-1635.

[26] T. Ide, H. Tsutsui, S. Kinugawa, N. Suematsu, S. Hayashidani, K. Ichikawa, H. Utsumi, Y. Machida, K. Egashira and A. Takeshita, Direct evidence for increased hydroxyl radicals originating from superoxide in the failing myocardium, Circulation Research 86 (2) (2000), $152-157$.

[27] H. Utsumi, E. Muto, S. Masuda and A. Hamada, In vivo ESR measurement of free radicals in whole mice, Biochem Biophys Research Communications 172 (1990), 1342-1348. [28] Y. Benjamini, A. M. Krieger and D. Yekutieli, Adaptive linear step-up procedures that control the false discovery rate, Biometrika 93 (2006), 491-507.

[29] V.E. Stefanov, B.F. Shchegolev, O.V. Kriyachko, N.V. Kuzmenko, S.V. Surma and I.M. Spivak, Model study of biological effects of weak static magnetic fields at the organismic and subcellular levels, Doklady Biological Sciences 461 (2015), 116-119. 
[30] O.K. Baskurt , R.A. Farley , H.J. Meiselman, Erythrocyte aggregation tendency and cellular properties in horse, human, and rat: a comparative study, American Journal of Physiology 273(6 Pt 2) (1997), H2604-12.

[31] H.G. Patel, R.V. Aras, K.R. Dave, S.S. Katyare, Kinetic attributes of $\mathrm{Na}^{+}, \mathrm{K}+$ ATPase and lipid/phospholipid profiles of rat and human erythrocyte membrane, Zeitschrift für Naturforschung C 55(9-10) (2000), 770-7.

[32] F. Kiss , E. Toth , K. Miszti-Blasius , N. Nemeth, The effect of centrifugation at various g force levels on rheological properties of rat, dog, pig and human red blood cells. Clinical Hemorheology and Microcirculation 62(3) (2016), 215-27.

[33] P. Kumar , P.K. Maurya, Epigallocatechin-3-Gallate Protects Erythrocyte Ca(2+)ATPase and $\mathrm{Na}(+) / \mathrm{K}(+)$-ATPase Against Oxidative Induced Damage During Aging in Humans, Advanced Pharmaceutical Bulletin 4(Suppl 1) (2014), 443-7.

[34] P. Singh, R.K. Kesharwani, K. Misra, S.I. Rizvi, The modulation of erythrocyte $\mathrm{Na}(+) / \mathrm{K}(+)$-ATPase activity by curcumin, Journal of Advance Research 6(6) (2015), 102330.

[35] S. Singh , K.B. Pandey, S.I. Rizvi, Erythrocyte senescence and membrane transporters in young and old rats, Archives of Physiology and Biochemistry 122(4), (2016), 228-234. [36] G. Garg , S. Singh , A.K. Singh , S.I. Rizvi, Metformin Alleviates Altered Erythrocyte Redox Status During Aging in Rats, Rejuvenation Research 20(1) (2017), 15-24.

[37] A.V. Domanski, E.A. Lapshina, I.B. Zavodnik, Oxidative processes induced by tert-butyl hydroperoxide in human red blood cells: chemiluminescence studies. Biochemistry (Moscow), 70 (7) (2005), 761-769.

[38] I. Sadowska-Woda I, B. Sychta, M. Rachel, E. Bieszczad-Bedrejczuk, Protective effect of desloratadine against oxidative stress in human erythrocytes in vitro. Environmental Toxicology and Pharmacology. 30 (2) (2010), 141-146. 
[39] Z. Liu, K. Han, Y. Lin, X. Luo, Antioxidative or prooxidative effect of 4hydroxyquinoline derivatives on free-radical-initiated hemolysis of erythrocytes is due to its distributive status. Biochimica et Biophysica Acta 1570 (2002), 97-103.

[40] T. N. Das, T. Dhanasekaran, Z. B. Alfassi, and P. Neta, Reduction Potential of the tertButylperoxyl Radical in Aqueous Solutions. Journal of Physical Chemistry A 102(1) (1998), 280-284.

[41] http://en.wikipedia.org/wiki/Tert-Butyl_hydroperoxide

[42] P.S. Rao and E. Hayon, Redox potentials of free radicals. IV. Superoxide and hydroperoxy radicals $\mathrm{O}^{2-}$ and HO2. Journal of Physical Chemistry A 79 (4) (1975), 397-402. [43] N. V. Goncharov, M.A. Terpilowski, A.D. Nadeev, I.V. Kudryavtsev, M.K. Serebriakova, V.P. Zinchenko, and P.V. Avdonin. Cytotoxic Power of Hydrogen Peroxide Effect on Endothelial Cells in vitro, Biochemistry (Moscow), Supplement Series A: Membrane and Cell Biology, 12 (2) (2018), 180-188. 


\section{Figure captions}

Fig. 1. Release of total $\mathrm{Hb}\left(\mu \mathrm{M}^{*}\right.$ in $0.2 \mathrm{ml}$ suspension with $5 \times 10^{11} \mathrm{RBC}$ ) into the supernatant of erythrocytes under exposure to EMF (48 $\mu \mathrm{T})$ or AMF $(0.192 \mu \mathrm{T})$, after 4 (a) and $24 \mathrm{~h}$ (b) h of incubation with various concentrations $(0-1500 \mu \mathrm{M})$ of TBHP. Each point is the mean of at least three replicates; error bars are not shown due to the scale inconvenience.

Fig. 2. Release of total (a), met- (b), and oxy- (c) hemoglobin ( $\mu \mathrm{M}$ in $0.2 \mathrm{ml}$ suspension with $5 \times 10^{11} \mathrm{RBC}$ ) into the supernatant of erythrocytes under exposure to EMF (48 $\left.\mu \mathrm{T}\right)$ or AMF $(0.192 \mu \mathrm{T})$ after $24 \mathrm{~h}$ of incubation in the absence of TBHP. ${ }^{*} \mathrm{p}<0.05 ; * * \mathrm{p}<0.01$.

Fig. 3. EPR spectra of the lysate of the erythrocyte suspension after $24 \mathrm{~h}$ incubation under EMF $(48 \mu \mathrm{T})$ or AMF $(0.192 \mu \mathrm{T})$ exposure, without TBHP (a) and with 100 (b) and $250 \mu \mathrm{M}$ (c) TBHP.

Fig. 4. Energy (a) and power (b) of EPR spectra of the erythrocyte suspension lysate after 24 h incubation under EMF (48 $\mu \mathrm{T})$ or AMF $(0.192 \mu \mathrm{T})$ exposure, without TBHP, and with 100, and $250 \mu \mathrm{M}$ (c) TBHP. 
(a)

4 hours

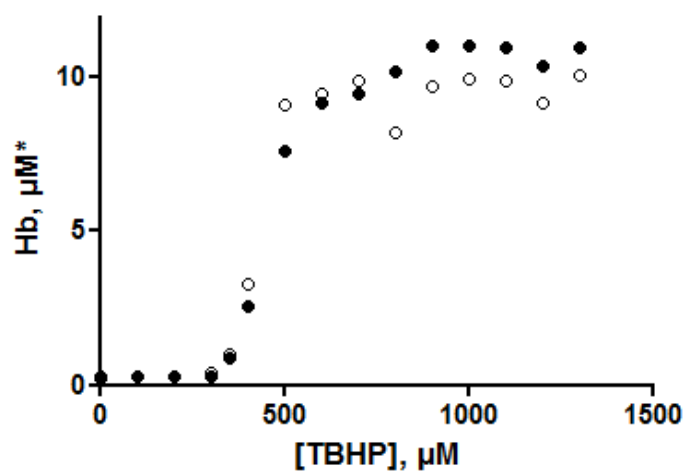

(b)

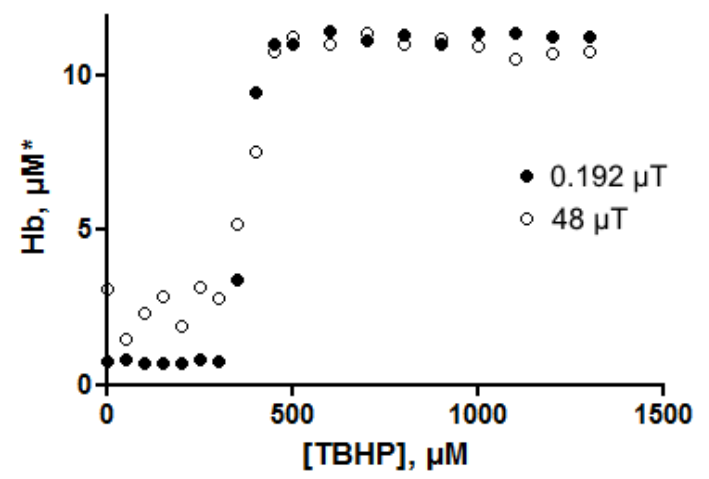

Fig. 1 

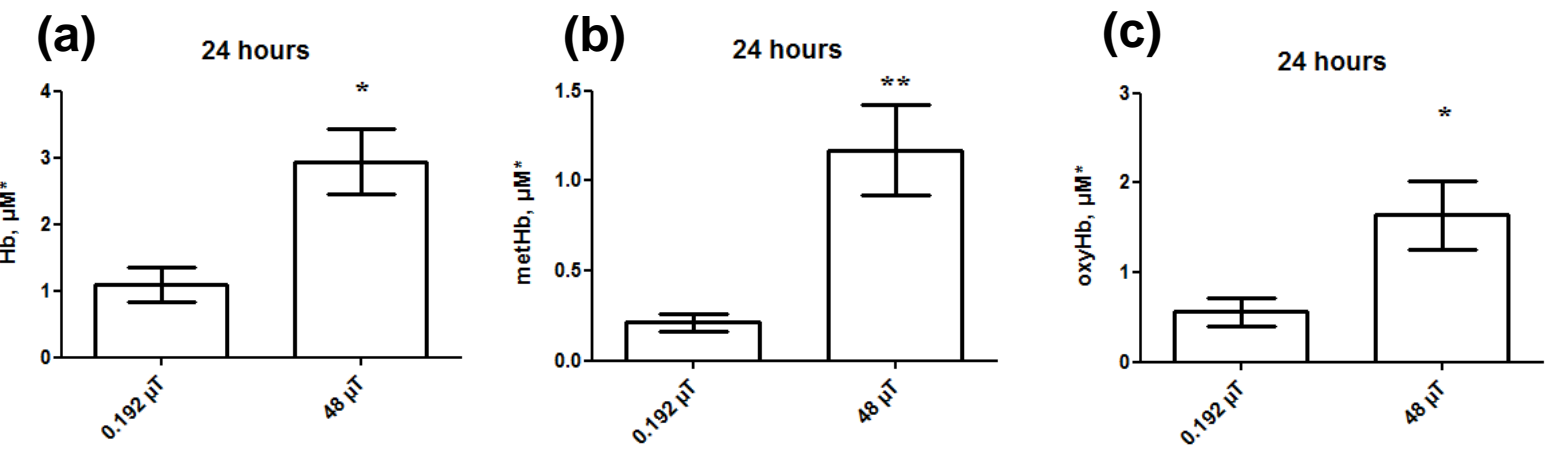

Fig. 2 
a)

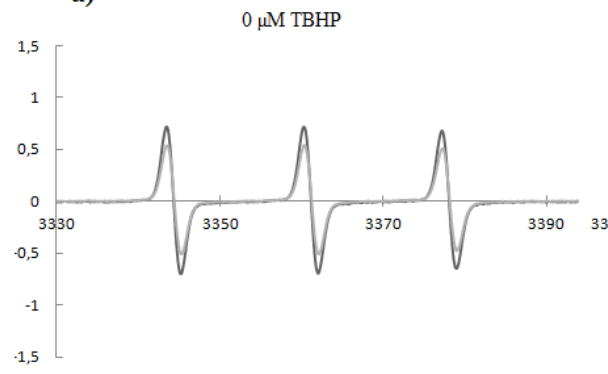

b)

$100 \mu \mathrm{M}$ TBHP

c)

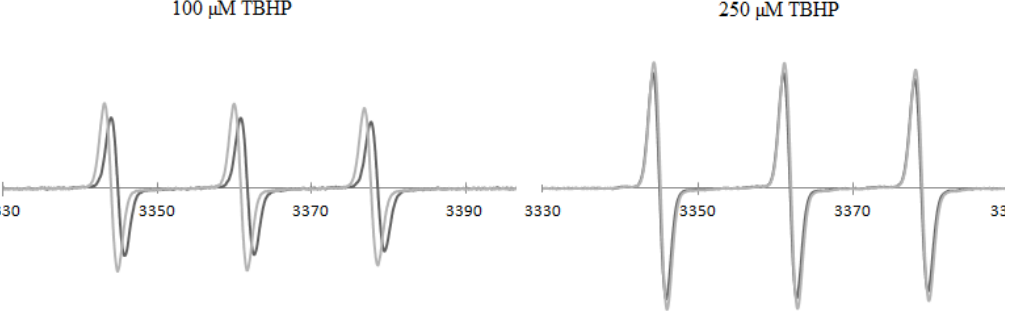

$-48 \mu \mathrm{T} \quad-0.192 \mu \mathrm{T}$

Fig. 3 

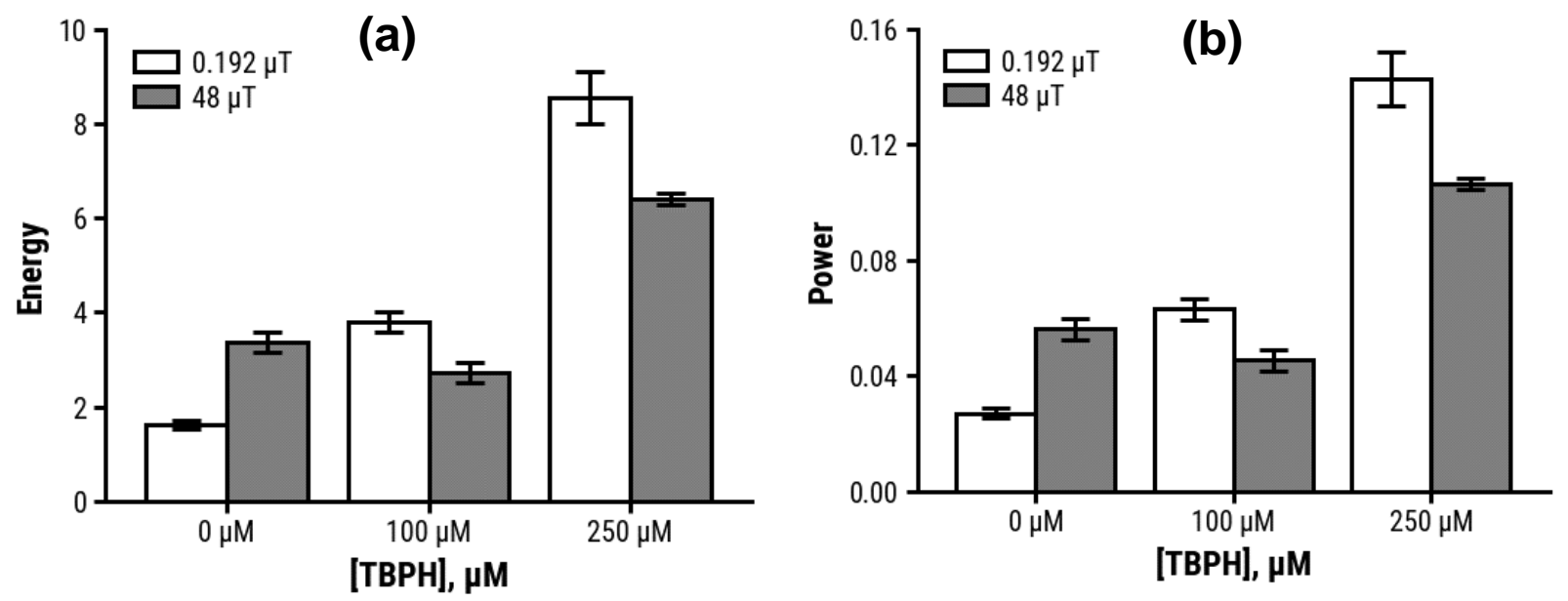

Fig. 4 